ARTICLE

DOI: $10.1038 / \mathrm{s} 41467-017-02804-6$

\title{
Room temperature magneto-optic effect in silicon light-emitting diodes
}

\author{
F. Chiodi', S.L. Bayliss ${ }^{2,3}$, L. Barast ${ }^{1,2}$, D. Débarre², H. Bouchiat ${ }^{2}$, R.H. Friend $0^{3}$ \& A.D. Chepelianskiii ${ }^{2}$
}

In weakly spin-orbit coupled materials, the spin-selective nature of recombination can give rise to large magnetic-field effects, e.g. on the electro-luminescence of molecular semiconductors. Although silicon has weak spin-orbit coupling, observing spin-dependent recombination through magneto-electroluminescence is challenging: silicon's indirect bandgap causes an inefficient emission and it is difficult to separate spin-dependent phenomena from classical magneto-resistance effects. Here we overcome these challenges and measure magneto-electroluminescence in silicon light-emitting diodes fabricated via gas immersion laser doping. These devices allow us to achieve efficient emission while retaining a welldefined geometry, thus suppressing classical magnetoresistance effects to a few percent. We find that electroluminescence can be enhanced by up to $300 \%$ near room temperature in a seven Tesla magnetic field, showing that the control of the spin degree of freedom can have a strong impact on the efficiency of silicon LEDs.

\footnotetext{
${ }^{1}$ Centre de Nanosciences et de Nanotechnologies, CNRS, Univ. Paris-Sud, Université Paris-Saclay, C2N-Orsay, Orsay 91405, France. ${ }^{2}$ Laboratoire de Physique des solides, CNRS, Univ. Paris-Sud, Université Paris-Saclay, LPS-Orsay, Orsay 91405, France. ${ }^{3}$ Cavendish Laboratory, University of Cambridge, J. J. Thomson Avenue, Cambridge CB3 OHE, UK. Correspondence and requests for materials should be addressed to

A.D.C. (email: alexei.chepelianskii@u-psud.fr)
} 
S pintronic effects in systems with weak spin-orbit coupling have attracted considerable attention due to their rich fundamental physics and potential for device applications ${ }^{1-}$ 4 . A class of these effects can be measured optically ${ }^{5-7}$, providing direct insight into phenomena such as spin-dependent recombination, where only the singlet state of an electron-hole pair can recombine radiatively back to the ground state. As external magnetic fields can change the spin statistics and energy levels in the sample, magneto-electroluminescence (MEL) effects have been seen as the hallmark of spin-dependent recombination phenomena and have given important insight into the role of spin in organic materials used for light-emitting diodes (LEDs) ${ }^{8-10}$. These spintronic effects can then be harnesseed to provide very senstive magnetic field sensors, sensible to external magentic fields of only a few mTesla comparable with the fluctuating hyperfine fields inside organic materials $s^{11-13}$ or to engineer new light-emitting device architectures through reverse intersystem crossing $^{14}$.

Similar to molecular semiconductors, silicon has weak spin-orbit coupling, but emission is much less efficient due to silicon's indirect bandgap, making analogous magneto-optic studies challenging and requiring careful engineering to prepare efficient LEDs ${ }^{15-19}$. In addition, observing spin-dependent MEL in silicon requires that the magnetic field and device currents are parallel to effectively suppress classical magnetoresistance (MR) contributions, which can enhance MR in silicon up to spectacular values even at room temperature ${ }^{18-22}$.

Here we address both of these challenges by developing a new fabrication method for efficient silicon LEDs (SiLEDs) using an original doping technique, gas immersion laser doping (GILD), and investigate spin-dependent recombination in SiLEDs. The GILD process ${ }^{23-26}$ allows us to reach doping levels well beyond the solubility threshold, which, as we describe below, gives rise to efficient emission, while retaining the well-defined planar geometry necessary to align electric and magnetic fields. Using our SiLEDs, we find that when classical MR effects are suppressed, EL can be substantially enhanced under a magnetic field near room temperature. We explain this phenomenon using a model of spindependent recombination ${ }^{27-29}$ of electron-hole pairs and use our analysis to estimate the exchange energy of weakly bound excitons in silicon. Our experiments provide an optoelectronic approach to probe the spin statistics of carriers in silicon-a material which is an excellent candidate for scalable spin quantum computing ${ }^{30-32}$. They also highlight the importance of controlling the spin degree of freedom for the efficiency of silicon light-emitting devices.

\section{Results}

Description of the system. We start by describing the fabrication procedure of the GILD SiLEDs (Fig. 1a) and the physical mechanism behind their enhanced efficiency, before discussing the MEL response of these devices. The Si LEDs were prepared by doping two $2 \times 2 \mathrm{~mm}^{2}$ spots with opposite polarities $p+/ n+$ on a n-Si [100] substrate of resistivity $45 \Omega \mathrm{cm}$ and thickness $700 \mu \mathrm{m}$ using the GILD technique (Fig. 1a). A precursor gas $\mathrm{PCl}_{3}\left(\mathrm{BCl}_{3}\right)$ for $n+(p+)$ doping is injected into an ultra-high vacuum chamber, where it saturates the chemisorbtion sites on the $\mathrm{Si}$ surface. The silicon is melted up to the desired doping depth by a XeCl $308 \mathrm{~nm}$ excimer laser of $25 \mathrm{~ns}$ pulse duration and tunable energy. This induces the rapid diffusion of the chemisorbed $\mathrm{B}$ into the liquid $\mathrm{Si}$ region (Fig. 1a). As a result, a box-like $\mathrm{Si}: \mathrm{P} / \mathrm{Si}: \mathrm{B}$ crystal is grown by liquid phase epitaxy on the underlying $\mathrm{Si}$ substrate ${ }^{25}$. Multiple chemisorbtion/melting cycles determine the dose of active dopants introduced in the doped layer. Due to the high recrystallization velocity $\left(4 \mathrm{~m} \mathrm{~s}^{-1}\right)$, the Si undergoes quenching, and high dopant concentrations beyond the solubility limit $\left(10^{20} \mathrm{~cm}^{-3} \text { for Si:B }\right)^{33}$ can be reached without introducing defects. In our experiments, we varied the doping concentration in the range $4.5 \times 10^{19}$ to $5 \times$ $10^{21} \mathrm{~cm}^{-3}$, while keeping the doping depth constant at $80 \mathrm{~nm}$ (pulse energy $\left.960 \mathrm{~mJ} \mathrm{~cm}^{-2}\right) . \operatorname{Ti}(15 \mathrm{~nm}) / \mathrm{Al}(200 \mathrm{~nm})$ electrodes were deposited on top of the doped spots after buffered hydrofluoric acid (BHF) deoxidation.

Device electroluminescence. We investigated EL from GILD SiLEDs in two different device geometries where the $p+$ and $n+$ spots were placed laterally, without overlap, on the same Si surface (Fig. 1b) and in a vertical geometry where the $n+/ p+$ were prepared on top of each other on opposite sides of the Si wafer (Fig. 1c). The alignment between top and bottom spots in the vertical geometry was achieved by looking through the silicon wafer with an infrared camera, which allowed to see the bottom GILD spots. This camera also allowed to record characterization a

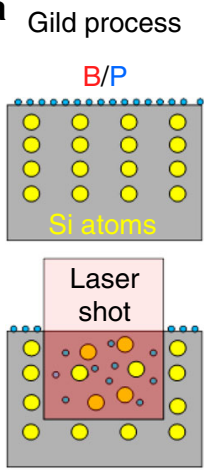

Si:B/Si:P

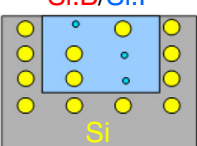

b Lateral SiLED
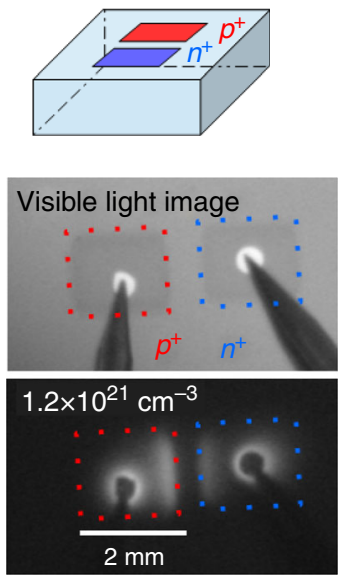

C
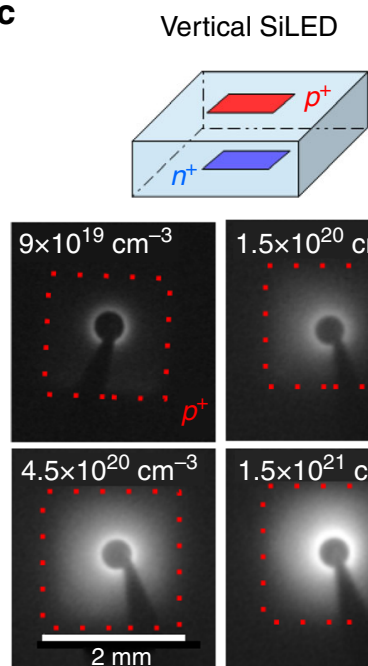

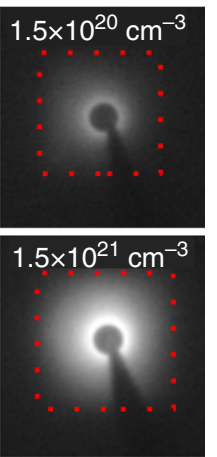

Fig. 1 Silicon light-emitting diodes from gas immersion laser doping (GILD). a Schematics of the GILD doping process: chemisorbtion of the dopant gas $\left(\mathrm{PCl}_{3} / \mathrm{BCL}_{3}\right)$; laser melting of the bulk Si and dopant incorporation in the liquid phase; Si:P or Si:B crystal epitaxy during solidification. $\mathbf{b}$ Schematic of lateral devices and infrared images of a silicon light-emitting device (SiLEDs) $\left(1.2 \times 10^{21} \mathrm{~cm}^{-3}\right)$ biased at $20 \mathrm{~mA}$ and at room temperature. $\mathbf{c}$ Schematic of vertical devices and infrared images of SiLEDs biased at $20 \mathrm{~mA}$ at room temperature for different doping levels $\left(9 \times 10^{19}, 1.5 \times 10^{20}, 4.5 \times 10^{20}, 1.5 \times 10^{21} \mathrm{~cm}^{-3}\right)$ 

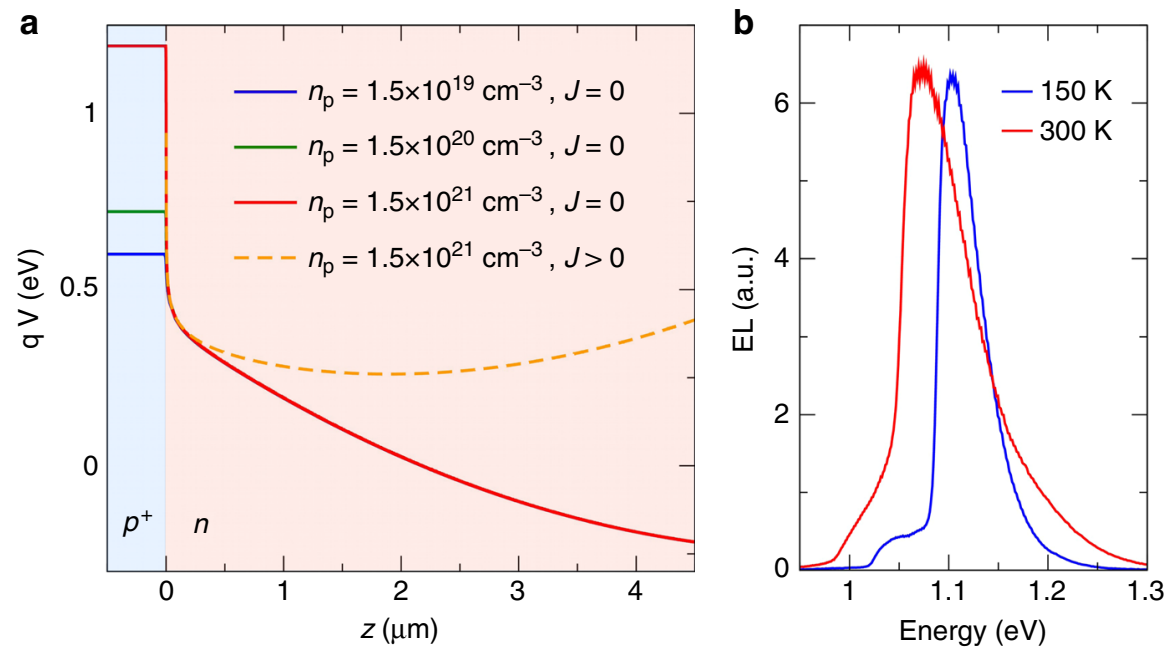

Fig. 2 Origin of enhanced emission in gas immersion laser doping silicon light-emitting devices. a Simulated electrostatic potential at the $p+/ n$ interface as a function of the vertical distance $z$ to the $p+/ n$ interface. Full curves show different $p+$ doping levels without external bias. The dashed curve shows the formation of a potential minimum under forward bias at a current density of $J=2 \times 10^{4} \mathrm{Am}^{-2}$ for the highest doping level. $\mathbf{b}$ Emission spectra from a lateral device with $1.2 \times 10^{21} \mathrm{~cm}^{-3}$ doping

images of EL devices. Images in the lateral geometry (Fig. 1b) show that EL occurs mainly at the heavily doped GILD spots on both $n+$ and $p+$ sides, whereas the undoped region in between the two spots remains dark. This shows that EL is enhanced near the $n+$ and $p+$ interfaces as compared with bulk Si. This observation is supported by the strong improvement of the device brightness as the GILD doping concentration increases (see Fig. 1c). The external quantum efficiency (EQE) for our brightest devices is around $0.05 \%$, which is comparable with the highest reported values for devices without anti-reflection treatment ${ }^{15}$.

The physical origin of the EL enhancement can be understood from the electrostatic profile within the devices, which we model using drift-diffusion simulations of the $p+/ n$ interface accounting for the Fermi statistics in the highly doped regions ${ }^{34}$. The simulated distributions of the electrostatic potential $V(z)$ are shown on Fig. 2a. In unbiased devices (see Supplementary Figure 1 for a more detailed discussion), the potential $V(z)$ inside the $n$ region $(z>0)$ is almost independent on the $p+$ doping concentration $n_{\mathrm{p}}$; however, a steep potential step forms at the $p+1$ $n$ interface, its height increasing with doping, creating a barrier that electrons have to overcome to leave the device. For parameters corresponding to the brightest devices the barrier is near an $\mathrm{eV}$ high and thermally activated transport is effectively prohibited. The $p+$ region thus has the role of an electron blocking layer, whereas the $n+$ region will similarly act as a hole blocking layer. Such layers are known to enhance the efficiency of organic LEDS ${ }^{35,36}$. When devices are biased the potential $V(z)$ remains constant in the $p+$ region and near the $p+/ n$ interface as the applied potential will mainly drop across the intrinsic weakly doped regions, which have much larger resistivity than the highly doped $p+$ region. A potential minimum therefore appears at sufficiently high forward bias near the $p+/ n$ and $n / n+$ interfaces. In these regions, located at a vertical distance of $1-2 \mu \mathrm{m}$ away from the interfaces, the internal electric field vanishes favoring radiative recombination, as a built-in electric field would otherwise drive electron-hole pairs apart. Similarly, the minority carriers (holes) predominantly recombine at the $n+$ interface as can be seen from the weaker EL observed from the $n+$ spot in lateral devices (see Fig. 1b). Finally, EL spectra in Fig. 2b (see also Supplementary Figure 2) are in very good agreement with previously reported lineshapes for bulk $\mathrm{Si}^{37-39}$ in agreement with

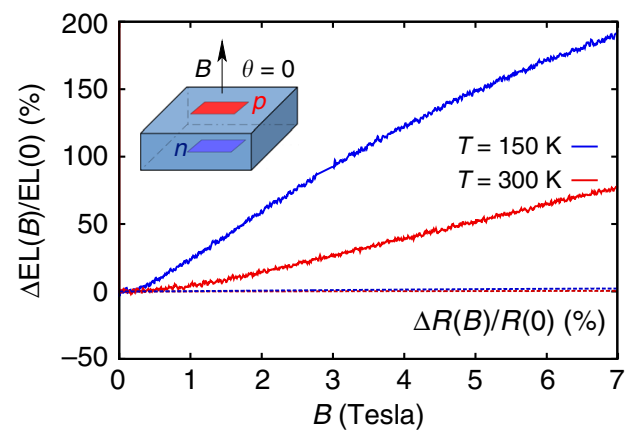

Fig. 3 Magneto-electroluminescence in silicon light-emitting devices. Comparison between MEL and MR effects in a vertical SiLED under a perpendicular magnetic field (magnetic field tilt angle $\theta=0$ relative to current lines) at 300 and $150 \mathrm{~K}$, where $\Delta \mathrm{EL}(B)=\mathrm{EL}(B)-\mathrm{EL}(0)$ and $\Delta R(B)=$ $R(B)-R(0)$. The $D C$ forward bias current was $10 \mathrm{~mA}$

our model predicting emission in the weakly doped Silicon a micrometer away from the $p+/ n$ and $n+/ n$ interfaces.

SiLED magneto-electroluminescence. Having described the structure of our devices and the physical mechanism behind their enhanced EL efficiency, we now use them to study the dependence of the EL on magnetic field in a vertical SiLED device (doping $3 \times 10^{21} \mathrm{~cm}^{-3}$ ); similar data were obtained on a device with $1.5 \times 10^{21} \mathrm{~cm}^{-3}$ doping. For this experiment, devices where mounted inside an optical access magnet (Oxford Instruments) and the EL was collected by a Ge photo-detector outside the cryostat. The SiLEDs were DC biased and the input of the Ge photodetector was chopped at $230 \mathrm{~Hz}$ to enhance sensitivity.

To study the role of spin in the MEL, we first applied a magnetic field $B$ perpendicular to the device surface, i.e., parallel to the internal electric field. We obtained a vanishing classical MR as shown in Fig. 3, where we observe only a weak residual MR in the $1 \%$ range both at 300 and $150 \mathrm{~K}$. The accuracy of this MR cancellation can seem surprising given the lack of electrical insulation around the spots. However, close to the onset voltage of the diode, the voltage drop mainly occurs accross the few 
a

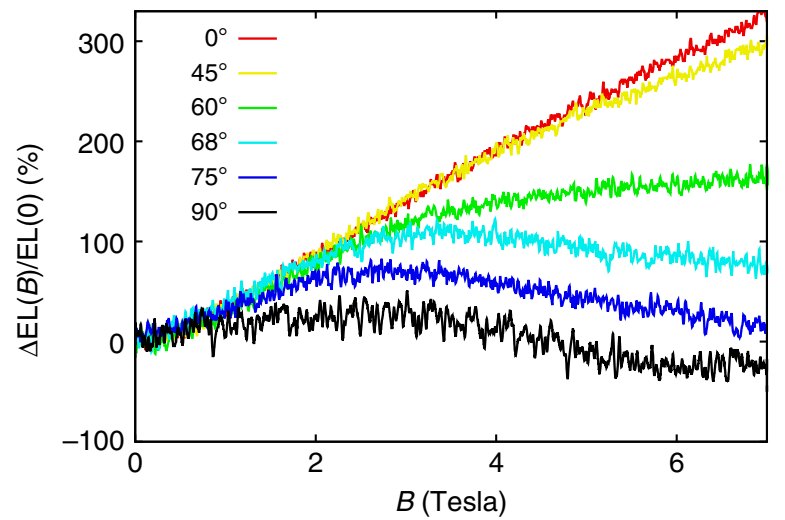

b

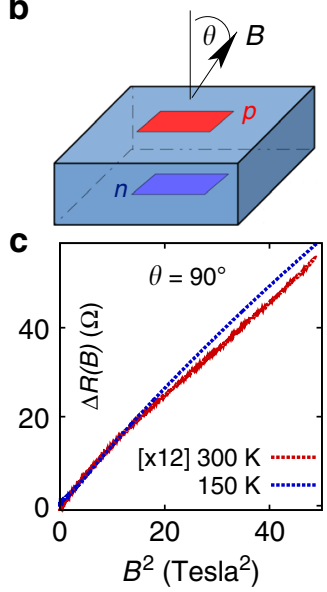

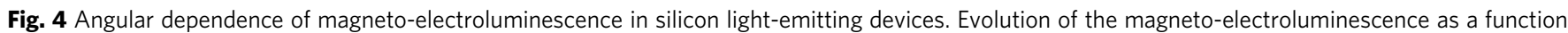
of the tilt angle $\theta$ between the magnetic field and the $5 \mathrm{~mA} \mathrm{DC}$ current at $150 \mathrm{~K}$ (data shown in a, experiment geometry is sketched in b). c MR response measured at $\theta=90^{\circ}$, which displays the classical $B^{2}$ dependence that contrasts with the MEL field dependence

micron wide depletion region between the $p+$ and $n$ regions (the extent of the depletion region is shown on Supplementary Figure 1) and the current lines cannot bend significantly considering the large spot size $2 \mathrm{~mm} \times 2 \mathrm{~mm}$. Figure 3 also plots the MEL response, which shows a drastically different behaviour. Compared with the MR signal, the EL exhibits a two orders of magnitude stronger dependence on the magnetic field, with $\Delta \mathrm{EL}$ $(B) / \mathrm{EL}(0)=75 \%$ at room temperature and an even higher $\Delta \mathrm{EL}$ $(B) / \mathrm{EL}(0)=290 \%$ value at $150 \mathrm{~K}$ (for a $5 \mathrm{~mA}$ current). The striking difference in magnitude of the MEL signal over the MR suggests that the magnetic field is increasing radiative recombination. As the EL quantum efficiency is low in silicon, a strong change in EL has little effect on the total current and hence the MR.

To further investigate the origin of the strong MEL effect in SiLEDs, we measured the MEL response as a function of the angle of the magnetic field relative to current lines Fig. 4. For moderate tilt angles $\theta$ below $45^{\circ}$, the MEL signal is nearly independent on the tilt. At larger tilt angles $\theta$ below $75^{\circ}$, the MEL is unchanged at low-magnetic fields but decreases from the low tilt angle behaviour at high magnetic fields. We attribute this decrease to the magneto-diode effect ${ }^{40-42}$ : the in-plane component of the magnetic field bends electron and hole trajectories so that they cross a larger distance through the device and thus have a higher non radiative-recombination probability. A significant bending of current lines can occur in perpendicular electric and magnetic fields when the ratio $\mu_{\mathrm{xy}} / \mu_{\mathrm{xx}}$ between Hall and longitudinal mobility is large. This quantity is around 1 at room temperature for $B=7 \mathrm{~T}$ and around 5 at $150 \mathrm{~K}$ (using the mobility values for a $45 \Omega \mathrm{cm}$ electron doped Si: $\mu_{\mathrm{xx}}=1.4 \times 10^{3} \mathrm{~cm}^{2} \mathrm{~V}^{-1} \mathrm{~s}^{-1}$ at room temperature and $\mu_{\mathrm{xx}}=7 \times 10^{3} \mathrm{~cm}^{2} \mathrm{~V}^{-1} \mathrm{~s}^{-1}$ at $\left.150 \mathrm{~K}\right)$. The negative magneto-diode contribution to MEL is further enhanced in lateral devices with longer current paths through the sample (see Supplementary Figure 4), with a negative MEL amplitude comparable with the ratio $\mu_{\mathrm{xy}} / \mu_{\mathrm{xx}}$.

The full MEL response is thus a superposition of a positive MEL component that depends only weakly on the $B$-field direction and a negative magneto-diode effect component that contributes more strongly at large tilt angles. A purely magnetodiode contribution would have the same $B$ dependence as the MR, which increases with the parallel magnetic field as $B^{2}$, as expected from the Drude law (see Fig. 4), in contrast with the observed MEL signal that displays a linear dependence with $B$, except at the lowest fields. As the angle and magnetic field dependence of the positive MEL effect are very different from the MR, we infer that the MEL is not determined by transport scattering times and may be related to spin degrees of freedom.

\section{Discussion}

The MEL effect observed in SiLEDs when the current and magnetic field are parallel $(\theta=0)$ can be explained as arising from the spin-dependent recombination of weakly bound electron-hole pairs within the devices, in analogy with the models developed by Kaplan et al. ${ }^{27}$ and Merrifield ${ }^{28}$. The ability of an electron-hole pair to recombine radiatively is determined by the overlap of the electron-hole pair wavefunction with the spin-zero singlet ground state-radiative recombination will only be efficient for electronhole pairs in an $S=0$ singlet configuration, the recombination from an $S=1$ triplet electron-hole pair being much less efficient (Fig. 5b). As the magnetic field is changed, the electron-hole eigenstates-which are in general not pure singlet or triplet spin states-are modified, altering their singlet overlap. As we explain below, this change in the electron-hole pair wavefunctions gives rise to a change in EL and can explain the lineshapes observed experimentally. Importantly, this effect is independent of the direction of the magnetic field and can therefore describe the angular-dependent MEL observations (Fig. 4). We note that although spin-dependent free-carrier recombination has previously been studied through circularly polarized emission ${ }^{43-45}$, here we invoke spin-dependent recombination of weakly bound exchange coupled electron-hole pairs to explain our MEL effects.

We start by considering the kinetic equation for the population of transient electron-hole pairs that are formed by brief collisions in the device recombination zone near room temperature. The population $X_{\mathrm{n}}$ of transient pairs with spin-eigenstates $|n\rangle$ is expected to follow the following rate equations:

$$
\dot{X}_{\mathrm{n}}=G_{\mathrm{n}}-\gamma_{\mathrm{s}} \alpha_{\mathrm{n}} X_{\mathrm{n}}-\gamma X_{\mathrm{n}} .
$$

Here, $G_{\mathrm{n}}$ is the electron-hole pair generation rate. The second term describes the probability of (spin-dependent) radiative recombination during collision events, with a rate $\gamma_{s}$. This term is proportional to the overlap of the electron-hole pair wavefunction with the spin-singlet state $\alpha_{\mathrm{n}}=|\langle S \mid n\rangle|^{2}$, where $|S\rangle$ is the singlet state. The final (spin-independent) $\gamma$ term reflects both the probability of escape from the shallow potential well where radiative recombination occurs in our devices and the rate of 

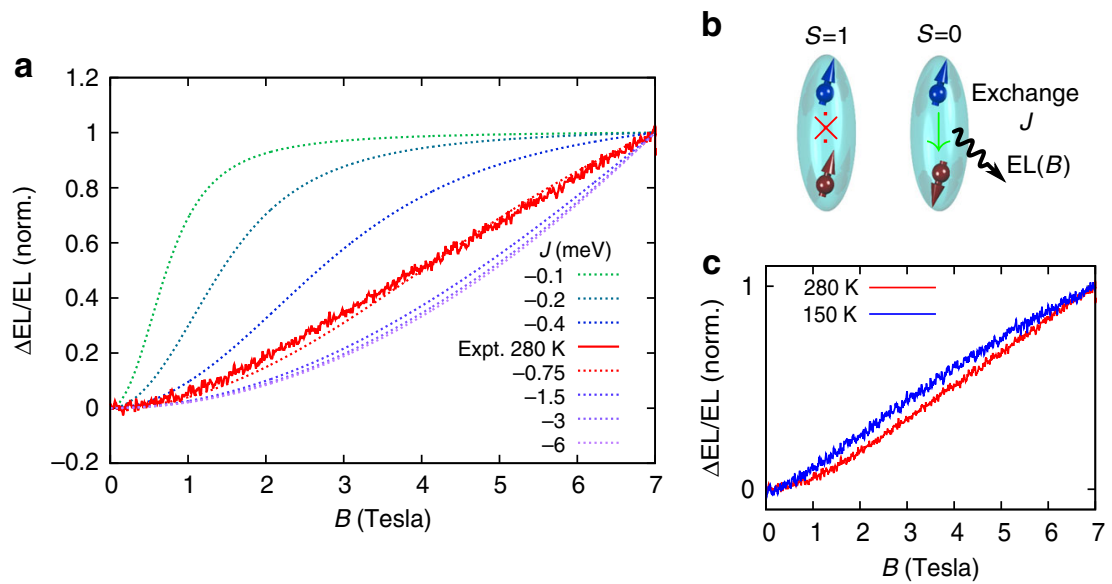

Fig. 5 Electron-hole spin-dependent recombination theory for the magneto-electroluminescence. Simulated magneto-electroluminescence using the model described in the text. Normalised simulations are shown in a for varying electron-hole exchange energy $J$ alongside the experimental data at $300 \mathrm{~K}$ (a comparison between normalised 300 and $150 \mathrm{~K}$ data is shown in $\mathbf{c}$ ). The characteristic saturation field of the magneto-electroluminescence is determined by the electron-hole exchange energy. Fitting to the $300 \mathrm{~K}$ experimental data gives an exchange energy $J=-0.75$ meV. Panel $\mathbf{b}$ illustrates the singlet and triplet spin pairings of a weakly bound electron/hole pair for which only the singlet state is emissive

nonradiative relaxation. Solving this in steady-state for the total emission from the electon-hole pairs EL $=\sum_{\mathrm{n}} \gamma_{\mathrm{s}} \alpha_{\mathrm{n}} X_{\mathrm{n}}$ we find

$$
\mathrm{EL} \propto \sum_{\mathrm{n}} \frac{G_{\mathrm{n}} \alpha_{\mathrm{n}}}{1+\varepsilon \alpha_{\mathrm{n}}},
$$

where $\varepsilon=\gamma_{s} / \gamma$. This sum depends non-linearly on the singlet projections $\left\{\alpha_{\mathrm{n}}\right\}$ and so a magnetic-field induced change in these can give rise to a change in emission. To compute EL from Equation 2, we calculate the singlet projections by diagonalizing the following electron-hole pair spin Hamiltonian

$$
\hat{H}=\underbrace{g_{\mathrm{e}} \mu_{\mathrm{B}} \mathbf{B} \cdot \hat{\mathbf{S}}_{\mathrm{e}}}_{\text {electron }}+\underbrace{g_{\mathrm{h}} \mu_{\mathrm{B}} \mathbf{B} \cdot \hat{\mathbf{S}}_{\mathrm{h}}+\lambda \hat{\mathbf{L}}_{\mathrm{h}} \cdot \hat{\mathbf{S}}_{\mathrm{h}}}_{\text {hole }}+\underbrace{J \hat{\mathbf{S}}_{\mathrm{e}} \cdot \hat{\mathbf{S}}_{\mathrm{h}}}_{\text {exchange }},
$$

where $g_{\mathrm{e}}, g_{\mathrm{h}}$ are electron and hole $g$-factors, $\mu_{\mathrm{B}}$ the Bohr magneton, $\mathbf{B}$ the applied field, $\hat{\mathbf{S}}_{\mathrm{e}}, \hat{\mathbf{S}}_{\mathrm{h}}$ are the electron and hole spins, $\lambda$ is the spin-orbit parameter for the hole with $\hat{\mathbf{L}}_{\mathrm{h}}$ the hole orbital angular momentum, and $J$ is the electron-hole exchange coupling. We note that the form of the effective spin Hamiltonians are similar for the transient interacting electron hole pairs considered here and tightly bound excitons, the main difference being the amplitude of the exchange interaction $J$ compared with temperature with $J \ll k_{\mathrm{B}} T$ for transient electon-hole pairs as opposed to bound excitons.

Figure 5 shows the change in EL as a function of magnetic field calculated from Equation 2 for various values of the electron-hole exchange parameter $J$, using $\lambda=-44 \mathrm{meV}^{46,47}, g_{\mathrm{e}}=g_{\mathrm{h}}=2^{48,49,50}$, Boltzmann populations, i.e., $G_{\mathrm{n}} \propto \mathrm{e}^{-E_{\mathrm{n}} / k_{\mathrm{B}} T}$ where $E_{\mathrm{n}}$ is the electron-hole pair energy, $k_{\mathrm{B}}$ is Boltzmann's constant, and $T=$ $300 \mathrm{~K}$ is the temperature. (This population distribution assumes that electron-hole pairs thermalize within their encounter time, but due to the high temperatures involved, similar results are obtained in the fully unpolarized limit where the generation rates are equal for each spin sublevel.) We note that for $\varepsilon=\gamma_{\mathrm{s}} / \gamma \lesssim 1$, which is the case we expect to apply here due to silicon's indirect bandgap, the lineshapes are independent of $\varepsilon$ and we set $\varepsilon=0.1$. This value should provide an upper bound for the internal quantum efficiency of our devices. The EQE we estimate is around $0.05 \%$, but taking into account the reflection due to the dielectric constant mismatch at the silicon interface, an upper bound $\varepsilon=0.1$ seems reasonable.
The simulations show an enhancement of the EL with magnetic field due to an increased number of states which overlap with the singlet ground state. This behavior arises from the fact that the spin-orbit interaction renormalises the effective hole $g$ factor (with effective Landé value $g_{\mathrm{h}} / 3$ for total spin $3 / 2$ holes), giving rise to a competition between the exchange interaction and the effective $g$-factor difference (and hence Zeeman energy) between electron and hole states. This leads to a mixing between singlet and triplet electron-hole spin configurations with a characteristic saturation field set by the competition between this Zeeman energy difference and the exchange term $B_{\text {sat }} \sim J / \Delta g_{\text {eff }} \mu_{\mathrm{B}}$. We find that the $300 \mathrm{~K}$ experimental lineshape can be reproduced with $J=-0.75 \mathrm{meV}$, which provides an estimate for typical exchange interactions for transient bound states formed during electron-hole collisions at room temperature in silicon; as expected, $J$ is smaller than the estimated exchange energy of 10 meV found for strongly bound excitonic states in Si nanocrystals ${ }^{51}$. Highlighting the importance of transient bound pairs, we show in the Supplementary Figure 3 that the positive MEL starts to decrease below $150 \mathrm{~K}$, a temperature that matches the exciton binding energy $(14.7 \mathrm{meV})$. This suggests that maximal sensitivity to magnetic field is achieved when the temperature is not too high, allowing interaction effects to show up, but not too small so that electron-hole encounter events do not result in irreversible binding. We emphasise that this model produces a MEL response, which does not depend on the direction of the external field, and can therefore explain the positive MEL component in our experiments (Fig. 4). Using our estimation for the exchange energy, we can check whether our assumption of weakly bound pairs is self-consistent. The typical size of the electron hole pair $d_{\mathrm{eh}}$ can be estimated by equating $J$ with the pair Coulomb energy $e^{2} / 4 \pi \varepsilon_{\mathrm{r}} \varepsilon_{0} d_{\mathrm{eh}}\left(\varepsilon_{\mathrm{r}}\right.$ is the dielectric constant of Silicon), leading to $d_{\mathrm{eh}}$ approximately equal to $160 \mathrm{~nm}$. For the pair to be well defined, the pair size has to be larger than the distance traveled by carriers due to thermal diffusion during the characteristic time-scale $\hbar / J$ of the interaction between the spins in the pair. With a thermal velocity $v_{\mathrm{t}}=10^{5} \mathrm{~m} \mathrm{~s}^{-1}$ for electrons at room temperature, the distance $\hbar v_{t} J^{-1}$ is around $80 \mathrm{~nm}$ and is indeed smaller than pair size. Although our model reproduces the observed MEL lineshapes, it fails to correctly reproduce the magnitude of the effect. The simulated value in Fig. 5 for $\Delta \mathrm{EL} / \mathrm{El}$ at a magnetic field $B$ of $7 \mathrm{~T}$ is around 0.1, as compared with the experimental 0.75 . 
Theoretical MEL can increase up to 0.76 in the $\varepsilon \gg 1$ limit but this would imply a very high internal quantum efficiency, which we do not believe to hold in our devices. Instead, we suggest that multiple recombination attempts, and a more detailed description of carrier kinetics, as well as electron-valley mixing can further amplify the theoretical MEL magnitude. Such a complete theory is beyond the scope of this work.

In conclusion, we have reported a strong increase in the brightness of SiLEDs under a magnetic field. These LEDs were fabricated using a novel technique, which allowed us to simultaneously suppress classical MR effects and obtain effective emission. In analogy with magneto-optic models developed for organic semiconductors, we explained our results as arising from the difference in recombination rates between singlet and triplet electron-hole pairs, allowing the electron-hole pair exchange energy to be estimated from the experimental lineshapes. Our investigations suggest an optoelectronic approach to probe spin transport properties in silicon near room temperature, a material with promise for quantum information processing and spintronics. They also show that the control of spin properties can allow to substantially increase the brightness of SiLEDs, which can be important components for chip to chip optical communication.

\section{Methods}

Gas immersion laser doping. The out-of-equilibrium laser doping was performed in ultra high vacuum $\left(10^{-9} \mathrm{mbar}\right)$ with a XeCl $308 \mathrm{~nm}$ excimer laser of pulse duration $25 \mathrm{~ns}$ and energy $0.96 \mathrm{~J} \mathrm{~cm}^{-2}$. The precursor gas used for the $\mathrm{B}(\mathrm{P})$ doping is $\mathrm{BCL}_{3}\left(\mathrm{PCL}_{3}\right)$. The doping level is finely controlled by the number of laser shots, each shot introducing a fixed $\mathrm{B}$ dose corresponding to the surface density of chemisorbtion sites for the precursor gas on the Si surface. The vertical alignment of the $n$ and $p$ doped spots is performed by illuminating the substrate from the bottom and observing the sample with an infrared camera, thus visualizing the bottom spot when doping the top one.

Magneto-electroluminescence. For magneto-electruminescence experiments the samples were mounted inside an optically accessible cryostat magnet providing a static magnetic field up to $7 \mathrm{~T}$. The samples were thermalized at the measurement temperature through a Helium vapor. The EL was collimated outside the cryostat and focused on a commercial Silicon or Germanium photodetector a meter away from the cryostat. Taking into account the higher sensitivity of Germanium photodetectors at the silicon emission wavelength the same results were obtained with the two types of photodetectors.

Data availability. The data and materials from this study are available on request from the corresponding author A.D.C.

Received: 8 March 2017 Accepted: 28 December 2017

Published online: 26 January 2018

\section{References}

1. Dyakonov, M. I. (ed.) Spin Physics in Semiconductors (Springer, 2008).

2. Jansen, R. Silicon spintronics. Nat. Mater. 11, 400-408 (2012).

3. Sun, Y. et al. Management of singlet and triplet excitons for efficient white organic light-emitting devices. Nature 440, 908 (2006).

4. Awschalom, D. D. \& Flatte, M. E. Challenges for semiconductor spintronics. Nat. Phys. 3, 153 (2007).

5. Schirhagl, R., Chang, K., Loretz, M. \& Degen, C. L. Nitrogen-vacancy centers in diamond: nanoscale sensors for physics and biology. Annu. Rev. Phys. Chem. 65, 83 (2014).

6. Nawrocki, M., Rubo, Yu. G., Lascaray, J. P. \& Coquillat, D. Suppression of the Auger recombination due to spin polarization of excess carriers and Mn 2+ ions in the semimagnetic semiconductor $\mathrm{Cd}_{0.95} \mathrm{Mn}_{0.05} \mathrm{~S}$. Phys. Rev. B(R.) 52, 2241 (1995).

7. Lepine, D. J. Spin-dependent recombination on silicon surface. Phys. Rev. B 6, 436 (1972).

8. Nguyen, T. D., Ehrenfreund, E. \& Vardeny, Z. V. Spin-polarized light-emitting diode based on an organic bipolar spin valve. Science 337, 204 (2012).
9. Shinar, J. Optically detected magnetic resonance studies of luminescencequenching processes in $\pi$ conjugated materials and organic lightemitting devices. Laser Photonics Rev. 6, 767 (2012).

10. Wang, J., Chepelianskii, A. D., Gao, F. \& Greenham, N. C. Control of exciton spin statistics through spin polarization in organic optoelectronic devices. Nat. Commun. 3, 1191 (2012).

11. Nguyen, T. D. et al. Isotope effect in spin response of [pi]-conjugated polymer films and devices. Nat. Mater. 9, 345 (2010).

12. Kersten, S. P., Schellekens, A. J., Koopmans, B. \& Bobbert, P. A. Magnetic-field dependence of the electroluminescence of organic light-emitting diodes: a competition between exciton formation and spin mixing. Phys. Rev. Lett. 106, 197402 (2011).

13. Wang, F., Macià, F., Wohlgenannt, M., Kent, A. D. \& Flatté, M. E. Phys. Rev. X 2, 021013 (2012).

14. Zhang, Q. et al. Efficient blue organic light-emitting diodes employing thermally activated delayed fluorescence. Nat. Photonics 8, 326 (2014).

15. Wai Lek Ng, et al. An efficient room-temperature silicon-based light-emitting diode. Nature 410, 192 (2001)

16. Green, M. A., Zhao, J., Wang, A., Reece, P. J. \& Gal, M. Efficient silicon lightemitting diodes. Nature 412, 805 (2001).

17. Saito, S. et al. Group IV light sources to enable the convergence of photonics and electronics. Front. Mater. 1, 66 (2014).

18. Delmo, M. P., Yamamoto, S., Kasai, S., Ono, T. \& Kobayashi, K. Large positive magnetoresistive effect in silicon induced by the space-charge effect. Nature 457, 1112 (2009).

19. Wan, C., Zhang, X., Gao, X., Wang, J. \& Tan, X. Geometrical enhancement of low-field magnetoresistance in silicon. Nature 477, 304 (2011).

20. Yang, D. et al. A large magnetoresistance effect in pn junction devices by the space-charge effect. Adv. Func. Mater. 23, 2918-2923 (2013).

21. Porter, N. A. \& Marrows, C. H. Linear magnetoresistance in $\mathrm{n}$-type silicon due to doping density fluctuations. Sci. Rep. 2, 565 (2012).

22. Schoonus, J. J. H. M., Bloom, F. L., Wagemans, W., Swagten, H. J. M. \& Koopmans, B. Extremely large magnetoresistance in boron-doped silicon. Phys. Rev. Lett. 100, 127202 (2008).

23. Boulmer, J., Guedj, C. \& Débarre, D. Incorporation of substitutional carbon in Si and SiGe by laser processing in methane and propylene Thin Solid Films 294, 137 (1997).

24. Bhaduri, A., Kociniewski, T., Fossard, F., Boulmer, J. \& Débarre, D. Optical and electrical properties of laser doped Si:B in the alloy range. Appl. Surf. Sc. 258, 9228 (2012).

25. Hoummada, K. et al. Absence of boron aggregates in superconducting silicon confirmed by atom probe tomography. Appl. Phys. Lett. 101, 182602 (2012).

26. Chiodi, F. et al. Laser doping for ohmic contacts in n-type Ge. Appl. Phys. Lett. 105, 242101 (2014).

27. Kaplan, D., Solomon, I. \& Mott, N. F. Explanation of the large spin-dependent recombination effect in semiconductors. J. De. Phys. Lett. 39, 51-54 (1978).

28. Merrifield, R. E. Magnetic effects on triplet exciton interactions. Pure Appl. Chem. 27, 481 (1971).

29. Bayliss, S. L., Greenham, N. C., Friend, R. H., Bouchiat, H. \& Chepelianskii, A. D. Spin-dependent recombination probed through the dielectric polarizability. Nat. Commun. 6, 8534 (2015).

30. Kane, B. E. A silicon-based nuclear spin quantum computer. Nature 393, 133-137 (1998).

31. Elzerman, J. M. et al. Single-shot read-out of an individual electron spin in a quantum dot. Nature 430, 431 (2004).

32. Tyryshkin, A. M. et al. Electron spin coherence exceeding seconds in highpurity silicon. Nat. Mater. 11, 143 (2012).

33. Murrell, A. J., Collart, E. J. H., Foad, M. A. \& Jennings, D. Process interactions between low-energy ion implantation and rapid-thermal annealing for optimized ultrashallow junction formation. J. Vac. Sci. Technol. B 18, 462 (2000).

34. Jüngel, A. Quasi-Hydrodynamic Semiconductor Equations. (Birkhauser, Berlin, 2001).

35. Ikai, M., Tokito, S., Sakamoto, Y., Suzuki, Y. \& Taga, Y. Highly efficient phosphorescence from organic light-emitting devices with an exciton-block layer. Appl. Phys. Lett. 79, 156 (2001).

36. Hagen, J. A., Li, W., Steckl, A. J. \& Grote, J. G. Enhanced emission efficiency in organic light-emitting diodes using deoxyribonucleic acid complex as an electron blocking layer. Appl. Phys. Lett. 88, 171109 (2006).

37. Tsybeskov, L., Moore, K. L., Hirschman, K. D., Hall, D. G. \& Fauchet, P. M. A Si-based lightemitting diode with roomtemperature electroluminescence at 1.1 eV. Appl. Phys. Lett. 69, 3411 (1996).

38. Chen, M.-J., Liang, E.-Z., Chang, S.-W. \& Lin, C.-F. Model for band-edge electroluminescence from metaloxidesemiconductor silicon tunneling diodes. J. Appl. Phys. 90, 789 (2001).

39. Lin, C.-F., Liu, C. W., Chen, M.-J., Lee, M. H. \& Lin, I. C. Electroluminescence at Si band gap energy based on metaloxidesilicon structures. J. Appl. Phys. 87, 8793-8795 (2000)

40. Popovic R. S. Hall Effect Devices (Institute of Physics Publishing, 2004). 
41. Stafeev, V. I. Modulation of diffusion length as a new principle of operation of semiconductor devices. Sov. Phys. Solid State 1, 763 (1959).

42. Cristoloveanu, S. The magnetodiode effect theory for the semiconductor and insulator regimes. Phys. Stat. Solid. (a) 64, 683-695 (1981).

43. Li, P. \& Dery, H. Theory of spin-dependent phonon-assisted optical transitions in silicon. Phys. Rev. Lett. 105, 037204 (2010).

44. Sircar, N. \& Bougeard, D. Experimental investigation of the optical spinselection rules in bulk $\mathrm{Si}$ and Ge/Si quantum dots. Phys. Rev. B 89, 041301(R) (2014).

45. Jonker, B. T., Kioseoglou, G., Hanbicki, A. T., Li, C. H. \& Thompson, P. E. Electrical spin-injection into silicon from a ferromagnetic metal/tunnel barrier contact. Nat. Phys. 3, 542 (2007).

46. Chuang S. L. Physics of Optoelectronic Devices - Wiley Series in Pure and Applied Optics (Wiley-Interscience Publication, 1995).

47. Madelung, O., Schulz, M., \& Weiss H. Landolt-Börnstein: Semiconductors Vol. 17 (Springer, 1982).

48. Zwanenburg, F. A., van Rijmenam, C. E., Fang, Y., Lieber, C. M. \& Kouwenhoven, L. P. Spin states of the first four holes in a silicon nanowire quantum dot. Nano Lett. 9, 1071 (2009).

49. Voisin, B. et al. Electrical control of g-factor in a few-hole silicon nanowire MOSFET. Nano Lett. 16, 88-92 (2015).

50. Harmon, N. J. \& Flatté, M. E. Spin relaxation in materials lacking coherent charge transport. Phys. Rev. B 90, 115203 (2014).

51. Brongersma, M. L., Kik, P. G., Polman, A., Min, K. S. \& Atwater, H. A. Sizedependent electron-hole exchange interaction in Si nanocrystals. Appl. Phys. Lett. 76, 351 (2000).

\section{Acknowledgements}

We thank M. Entin, L. Magaril, A. Rowe, R. Colombelli and T. Chanelière for fruitful discussions and acknowledge support from PICS-Royal society, ANR SPINEX, and ANR SUPERR.This work is supported by public grants overseen by the French National Research Agency (ANR) as part of the "Investissements d'Avenir" program (reference: ANR-10-LABX-0035, Labex NanoSaclay, project 16HG1457 and ANR-10-LABX-0039 PALM).

\section{Author contributions}

F.C., S.L.B., L.B., D.D., H.B., R.H.F. and A.D.C. all participated in magneto-EL measurements and spectroscopic characterization of the samples. F.C., L.B. and D.D. fabricated the SiLEDs. S.L.B., H.B., R.H.F. and A.D.C. developed the theoretical model.

\section{Additional information}

Supplementary Information accompanies this paper at https://doi.org/10.1038/s41467017-02804-6.

Competing interests: The authors declare no competing financial interests.

Reprints and permission information is available online at http://npg.nature.com/ reprintsandpermissions/

Publisher's note: Springer Nature remains neutral with regard to jurisdictional claims in published maps and institutional affiliations.

\begin{abstract}
(c) (i) Open Access This article is licensed under a Creative Commons Attribution 4.0 International License, which permits use, sharing, adaptation, distribution and reproduction in any medium or format, as long as you give appropriate credit to the original author(s) and the source, provide a link to the Creative Commons license, and indicate if changes were made. The images or other third party material in this article are included in the article's Creative Commons license, unless indicated otherwise in a credit line to the material. If material is not included in the article's Creative Commons license and your intended use is not permitted by statutory regulation or exceeds the permitted use, you will need to obtain permission directly from the copyright holder. To view a copy of this license, visit http://creativecommons.org/ licenses/by/4.0/.
\end{abstract}

(C) The Author(s) 2018 